\title{
THE PLANETARY NEBULA LUMINOSITY FUNCTION (PNLF)
}

\section{OF OUR GALAXY}

\section{COMPARED TO THOSE OF OTHER GALAXIES}

\author{
R. H. MÉNDEZ \\ University Observatory, Munich, Germany
}

Nowadays it is possible to detect planetary nebulae, and measure their [O III] $\lambda 5007$ fluxes, in many galaxies. Given this information it is relatively straightforward to build the [O III] $\lambda 5007$ PNLF for any galaxy, because we can always assume all its PNs to be essentially at the same distance from us.

Of course the situation is much more difficult for PNs in the solar neighborhood. We have two problems here: (1) the individual PN distances are poorly known, except for $\sim 25$ objects; (2) in the literature there is no agreement on which are the 25 PNs with reliable distances. Clearly it was a wise decision to calibrate the PNLF method of extragalactic distance determinations using the distance to $M 31$ (Ciardullo et al. 1989, Jacoby et al. 1992). In these short notes I review if it may be possible to build a PNLF for our Galaxy (the answer is: yes, probably more than one) and discuss if it would be worth the trouble (the answer is: yes, it would) and what can be done to achieve that elusive goal.

Obviously we cannot build our Galactic PNLF using statistical distances obtained through assumptions (explicit or implicit) about recombination line fluxes like $\mathrm{H} \beta$. For example, the distances recently obtained by Cahn et al. (1992) using the Daub (1982) scheme. In this scheme all nebulae are forced to follow the same relation between ionized mass and optical thickness parameter $T$, which has the effect of artificially eliminating the dispersion in the values of the absolute radio and $\mathrm{H} \beta$ fluxes. A plot of the $\mathrm{H} \beta$ PNLF obtained with the Cahn et al. (1992) distances shows a vertical PNLF bright end, which is clearly an artifact of the method. In summary, we must avoid distances which would deform the very PNLF we want to build.

What about the sample of PNs in our Galactic bulge? The Galactic bulge PNLF has been compared to those of M 31 and the LMC by Pottasch (1990). He assumed that the PNLF is universal, and derived a reasonable distance to the Galactic center. However, now we are more interested in testing the universality of the PNLF. Numerical simulations of the PNLF (Méndez et al. 1993) have shown that we should expect the observed bright end of the PNLF to be a function of (1) the size of the PN sample, (2) the maximum final post-AGB mass in the corresponding stellar population, (3) how optically thin are the brightest PNs in the sample. From this work (and especially from the second point of the three listed above) we infer that we should not expect, for example, the PNLF in the Galactic bulge to be necessarily the same as the PNLF in the solar neighborhood. The local PN sample corresponds to a population which can be assumed to have a more or less constant star formation rate, while substantial star formation in the bulge has probably ceased at least 5 Gyr ago, producing at the present time a smaller turnoff mass, therefore a smaller maximum final post-AGB mass, and consequently a fainter PNLF (more details in 
Méndez et al. 1993). The difference between the bright ends of the local and bulge PNLFs might be as large as 0.4 mags.

Thus we see that it would be unsatisfactory to derive local PN distances by assuming a universal PNLF, and also that it would be very instructive to confirm if the bright end of the PNLF is indeed somewhat brighter for PNs in the solar neighborhood than for PNs in our Galactic bulge and the bulge of M 31 .

In order to detect differences of about 0.4 mags in the bright ends of these PNLFs, we need individual PN distances with errors below $25 \%$. My favorite method is to derive spectroscopic distances from NLTE model atmosphere studies of the central stars (e.g. Méndez et al. 1992). These distances are rejected by some authors because they are somewhat larger than distances determined with other methods. However, there are reasons to believe that the other distances are too small. Let me illustrate this point using the distances of Cahn et al. (1992). Let us call them CKS distances. This large set of statistical distances was calibrated with 19 distances considered by Cahn et al. to be well known: 15 reddening distances, 2 spectroscopic parallaxes of cooler companions and 2 cluster distances (the NLTE spectroscopic distances were not included in their calibration).

If we plot each CKS distance as a function of the corresponding NLTE spectroscopic distance, for 21 objects in common, we find a not so bad correlation coefficient, 0.89 . The linear regression has a slope of 0.84 , which implies that the NLTE spectroscopic distances are about $20 \%$ larger.

But now we can make a test: it turns out that 128 of the CKS distances correspond to PNs within 10 degrees of the Galactic center. Rejecting 16 objects with angular diameters larger than 10 arc seconds (most probably foreground PNs) we are left with 112 , of which $80 \%$ or $90 \%$ are expected to be physically close to the Galactic center. The distance to the Galactic center is $8.0 \pm 0.5 \mathrm{kpc}$ (Reid 1993). A histogram with the number of CKS distances at each distance, for these 112 PNs, shows a maximum between 6 and $7 \mathrm{kpc}$. Only 30 of these distances are larger than 8 kpc. If Cahn et al. had calibrated their distances with the NLTE spectroscopic distances, which are $20 \%$ larger, they would have obtained a more satisfactory distance to the Galactic center. It is plausible that some of the 15 reddening distances used for their calibration are too small.

What happens if we try to build the local PNLF using the NLTE spectroscopic distances? This was done by Méndez et al. (1993), who obtained a fainter bright end than that of $M 31$, the reason being most probably the very small size of the local sample. After adding a dozen of other selected individual distances, the bright end of the local PNLF became somewhat brighter, but the sample size was still too small to derive any reliable conclusion. It is clear, anyway, that there is no need to decrease the spectroscopic distances.

In the near future it may be possible to increase the number of NLTE spectroscopic distances to more than 50, perhaps even more than 100 . However, most of the PNs will be rather faint. We would need a method to select the most promising candidates to populate the bright end of the PNLF. One possibility is a method of distance determinations recently presented by Zhang (1993, see also Zhang and Kwok 1993). I have used their method, after modifying some input data in 30 cases, to produce a $\lambda 5007$ PNLF for 125 local PNs. The bright end of this PNLF shows 
6 PNs which are brighter than the brightest PN observed in M 31: NGC 3211, NGC 6302, IC 2165, IC 2501, IC 5117, Me 2-2. Unfortunately the uncertainties in the distances are sufficiently large $( \pm 40 \%)$ to make this result inconclusive for the comparison of local vs. bulge populations (since there is a cutoff at the bright end, errors in the distances will tend to shift a few PNs to very bright fluxes). But the central stars of these 6 PNs deserve detailed NLTE spectroscopic studies, in order to verify their distances and confirm if the nebular $\lambda 5007$ fluxes are indeed so high.

To build a PNLF for the Galactic bulge is easier, but the interpretation is also difficult. From the Catalogue of Galactic PNs (Acker et al. 1992) we can select PNs within 10 degrees of the Galactic center, with angular diameters smaller than 10 arc seconds and reliable radio fluxes (which should give better results than $\mathrm{H} \beta$ fluxes, in view of the high reddening). There are 141 such objects. Adopting a common distance of $8 \mathrm{kpc}$, we easily obtain the $\mathrm{H} \beta$ PNLF, and we find several "superbright" PNs: Hb 4, Hb 6, M1-40, M1-41. The difficulty in the interpretation comes from the fact that we should expect, among the 141 objects, something like 20 in the foreground, and it is not necessarily surprising to find 4 of them populating the PNLF bright end. Now obviously we cannot simply assume that these 4 are foreground PNs, because we would be running in circles. What we need is independent and accurate distance determinations for these 4 PNs. An effort to obtain NLTE spectroscopic distances for these central stars would probably help to better define the bright end of our bulge PNLF.

In conclusion: to speak about "the PNLF of our Galaxy" is misleading, because we have some reasons to believe that different populations in our Galaxy should produce slightly different PNLFs. The information currently available is not sufficient to confirm if such differences really exist; but I have tried to indicate a few observational projects which might help us to obtain a clearer picture. These problems are not likely to affect the use of the PNLF for extragalactic distance determinations, because, in order to avoid contamination of the PNs with massive (Population I) $\mathrm{H}$ II regions, the PNLF method has always been applied to populations without recent star formation (with the exception of the LMC, whose PNLF also gives unclear results which I cannot discuss here). But a better knowledge of the PNLF in the solar neighborhood would give us more confidence in modeling and using the PNLF as a tool for studies of the initial-final mass relation and related mass loss processes in a variety of stellar populations.

\section{References}

Acker A., Ochsenbein F., Stenholm B. et al. 1992, Catalogue of Galactic PNs, E.S.O.

Cahn J.H., Kaler J.B., Stanghellini L. 1992, A\&AS 94, 399

Ciardullo R., Jacoby G.H., Ford H.C., Neill J.D. 1989, ApJ 339, 53

Daub C.T. 1982, ApJ 260, 612

Jacoby G.H., Branch D., Ciardullo R. et al. 1992, PASP 104, 599

Méndez R.H., Kudritzki R.P., Herrero A. 1992, A\&A 260, 329

Méndez R.H., Kudritzki R.P., Ciardullo R., Jacoby G.H. 1993, A\& A 275, 534

Pottasch S.R. 1990, A\&A 236, 231

Reid M.J. 1993, ARA\&A 31, 345

Zhang C.Y. 1993, ApJ 410, 239

Zhang C.Y., Kwok S. 1993, ApJS 88, 137 